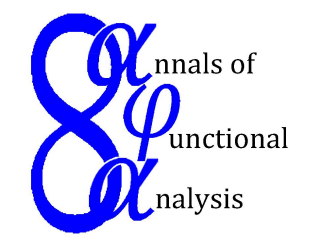

Ann. Funct. Anal. 6 (2015), no. 3, 110-117

http://doi.org/10.15352/afa/06-3-10

ISSN: 2008-8752 (electronic)

http://projecteuclid.org/afa

\title{
NOTE ON $(m, q)$-ISOMETRIES ON AN HYPERSPACE OF A NORMED SPACE
}

\author{
ANTONIO MARTINÓN \\ Communicated by V. Müller
}

\begin{abstract}
Given a normed space $X$ we consider the hyperspace $k(X)$ of all non-empty compact convex subsets of $X$ endowed with the Hausdorff distance. We prove that if $T: X \longrightarrow X$ is an $(m, q)$-isometry, then it is possible that the map $k(T): k(X) \longrightarrow k(X), k(T) C:=T C$, is not an $(m, q)$-isometry. Moreover, if $\widehat{k(X)}$ is the Rådström space associated to the hyperspace $k(X)$, then $\mathcal{T}: k(X) \longrightarrow k(X)$ is an $(m, q)$-isometry if and only if $\widehat{\mathcal{T}}: \widehat{k(X)} \longrightarrow \widehat{k(X)}$ is an $(m, q)$-isometry.
\end{abstract}

\section{INTRODUCTION}

Throughout this paper, $X$ is a real normed space and $\|\cdot\|$ its norm, $L(X)$ the class of all bounded linear operators $T: X \longrightarrow X, m$ a positive integer and $q$ a positive real number, unless stated otherwise.

The notion of $(m, q)$-isometry in the setting of metric spaces was introduced in [3]: a map $T: E \longrightarrow E$, on a metric space $E$ with distance $d$, is called an $(m, q)$-isometry if

$$
\sum_{i=0}^{m}(-1)^{m-i}\left(\begin{array}{c}
m \\
i
\end{array}\right) d\left(T^{i} x, T^{i} y\right)^{q}=0 \quad(x, y \in E) .
$$

An $(m, q)$-isometry is called strict whenever is not an $(m-1, q)$-isometry. Of course, the $(1, q)$-isometries are the isometries. This definition generalizes the concept of $m$-isometry firstly introduced on Hilbert spaces by J. Agler [1]. Some time after the notion of $(m, q)$-isometry on Banach spaces was defined by Bayart [2] and Sid Ahmed [7].

Date: Received: Dec. 15, 2014; Accepted: Jan. 14, 2015.

2010 Mathematics Subject Classification. Primary 54E40; Secondary 47B99.

Key words and phrases. Rådström space, $m$-isometry, hyperspace, wighted shift operator. 
In [4] it was introduced a notion of $m$-isometry on certain hyperspaces of a Banach space. In this paper we study $(m, q)$-isometries on the hyperspace $k(X)$ of all non-empty convex compact subsets of a normed space $X$. Given an operator $T \in L(X)$ we consider the map $k(T): k(X) \longrightarrow k(X)$, defined by $k(T) C:=T C$. It is possible that $T$ is an $(m, q)$-isometry but $k(T)$ is not an $(m, q)$-isometry. More precisely, we prove that any weighted shift operator $S_{w} \in L\left(\ell_{2}\right)$ which is a $(2,2)$-isometry induces a map $k\left(S_{w}\right): k\left(\ell_{2}\right) \longrightarrow k\left(\ell_{2}\right)$ which is not an $(2,2)$ isometry.

Using a construction by Rådström we associate to $k(X)$ the normed space $\widehat{k(X)}$, being $k(X)$ a subspace of $\widehat{k(X)}$. We prove that $\mathcal{T}: k(X) \longrightarrow k(X)$ is an $(m, q)$-isometry if and only if $\widehat{\mathcal{T}}: \widehat{k(X)} \longrightarrow \widehat{k(X)}$ is an $(m, q)$-isometry.

\section{The HYPERSPACE $k(X)$}

Given a real normed space $X$, we consider the hyperspace

$$
k(X):=\{C \subset X: \emptyset \neq C \text { compact convex }\} .
$$

For $C, D \in k(X)$ and $\alpha$ scalar, we write $C+D:=\{x+y: x \in C, y \in D\}$ and $\alpha C:=\{\alpha x: x \in C\}$. Some properties of the class $k(X)$ are given in the following proposition:

Proposition 2.1. For $C, D, E \in k(X) ; \lambda, \mu \geq 0$ and $\alpha$ scalars,

(1) $C+D \in k(X)$

(2) $(C+D)+E=C+(D+E)$ and $C+D=D+C$

(3) $C+E=D+E \Longrightarrow C=D$

(4) $\alpha C \in k(X)$

(5) $\alpha(C+D)=\alpha C+\alpha D$ and $(\lambda+\mu) C=\lambda C+\mu C$

Proof. The property (3) is [6, Lemma 2]. The other properties are simple.

We introduce the norm of $C \in k(X)$ :

$$
\|C\|:=\sup _{x \in C}\|x\| \text {. }
$$

Proposition 2.2. For $C, D \in k(X)$ and $\alpha$ scalar,

(1) $\|C\|=0 \Longleftrightarrow C=\{0\}$

(2) $\|C+D\| \leq\|C\|+\|D\|$

(3) $\|\alpha C\|=|\alpha|\|C\|$

Proof. Routine.

The class $k(X)$ is endowed with the Hausdorff distance $h$ : given $C, D \in k(X)$, we put

$$
h(C, D):=\inf \left\{\varepsilon>0: C \subset D+\varepsilon B_{X} \text { and } D \subset C+\varepsilon B_{X}\right\},
$$

where $B_{X}$ is the unit closed ball of $X$. In the next result we collect some basic facts about the distance $h$.

Proposition 2.3. For $C, D, E \in k(X)$ and $\alpha$ scalar, 
(1) $h$ is a metric on $k(X)$; moreover, if $X$ is a Banach space, then $k(X)$ is complete.

(2) $h(C+E, D+E)=h(C, D)$

(3) $h(\alpha C, \alpha D)=|\alpha| h(C, D)$

(4) $h(C,\{0\})=\|C\|$

Proof. The property (1) is well known and (4) is clear. In order to prove (2), notice that, for every $\varepsilon>0$, we can write

$$
\begin{aligned}
h(C+E, D+E)<\varepsilon & \Longrightarrow C+E \subset D+E+\varepsilon B_{X} \text { and } D+E \subset C+E+\varepsilon B_{X} \\
& \Longrightarrow C \subset D+\varepsilon B_{X} \text { and } D \subset C+\varepsilon B_{X} \\
& \Longrightarrow h(C, D) \leq \varepsilon,
\end{aligned}
$$

by Proposition 2.1 (3). Analogously, $h(C, D)<\varepsilon \Longrightarrow h(C+E, D+E) \leq \varepsilon$. Therefore, (2) is true.

Now we prove (3). We have that the equality is obvious if $\alpha=0$. Assume $\alpha \neq 0$. Then

$$
\begin{aligned}
h(\alpha C, \alpha D)<\varepsilon \Longrightarrow & \alpha C \subset \alpha D+\varepsilon B_{X} \text { and } \alpha D \subset \alpha C+\varepsilon B_{X} \\
\Longrightarrow & C \subset D+\alpha^{-1} \varepsilon B_{X}=D+|\alpha|^{-1} \varepsilon B_{X} \\
& \text { and } D \subset C+\alpha^{-1} \varepsilon B_{X}=C+|\alpha|^{-1} \varepsilon B_{X} \\
\Longrightarrow & h(C, D) \leq|\alpha|^{-1} \varepsilon \\
\Longrightarrow & |\alpha| h(C, D) \leq \varepsilon .
\end{aligned}
$$

Analogously, $|\alpha| h(C, D)<\varepsilon \Longrightarrow h(\alpha C, \alpha D) \leq \varepsilon$. Consequently, (3) holds.

Observe that the property (2) in the above proposition depends on the fact that $E$ is bounded and that both sets $C+\varepsilon B_{X}$ and $D+\varepsilon B_{X}$ are convex closed, since $C$ and $D$ are convex compact (see [6, Lemmas 2 and 3]).

It is obvious that we can identify $X$ with $\{\{x\}: x \in X\} \subset k(X)$. For $x, y \in X$ and $\alpha$ scalar we have that $\{x\}+\{y\}=\{x+y\}, \alpha\{x\}=\{\alpha x\}$ and $h(\{x\},\{y\})=$ $\|x-y\|$. Notice that, in general,

$$
h(C, D) \leq\|C-D\| \quad(C, D \in k(X))
$$

and it is possible that $h(C, D)<\|C-D\|$. For example, $h(C, C)=0<\|C-C\|$ whenever $C$ is not a singleton.

\section{MAPS ON $k(X)$}

We say that a map $\mathcal{T}: k(X) \longrightarrow k(X)$ is linear if, for $C, D \in k(X)$ and $\alpha$ scalar,

$$
\mathcal{T}(C+D)=\mathcal{T} C+\mathcal{T} D \quad \text { and } \quad \mathcal{T}(\alpha C)=\alpha \mathcal{T} C
$$

Given $\mathcal{T}: k(X) \longrightarrow k(X)$ linear we define the norm of $\mathcal{T}$ by

$$
\|\mathcal{T}\|=\sup _{\{0\} \neq C \in k(X)} \frac{\|\mathcal{T} C\|}{\|C\|}=\sup _{C \in k(X),\|C\|=1}\|\mathcal{T} C\|
$$

Hence, for every $C \in k(X)$, we have that $\|\mathcal{T} C\| \leq\|\mathcal{T}\|\|C\|$. We say that $\mathcal{T}$ is bounded if $\|\mathcal{T}\|<\infty$. 
The following results are very similar to analogous facts about linear operators between normed spaces and we omit the proof.

Proposition 3.1. Let $\mathcal{T}: k(X) \longrightarrow k(X)$ a linear map. The following assertions are equivalent:

(1) $\mathcal{T}$ is uniformly continuous

(2) $\mathcal{T}$ is continuous

(3) $\mathcal{T}$ is continuous at $\{0\}$

(4) There exists $M>0$ such that, for every $C \in k(X),\|\mathcal{T} C\| \leq M\|C\|$

(5) $\mathcal{T}$ is bounded

We denote by $L(k(X))$ the class of all bounded linear maps $\mathcal{T}: k(X) \longrightarrow k(X)$.

Proposition 3.2. For $\mathcal{T}, \mathcal{S} \in L(k(X))$ and scalar $\alpha$,

(1) $\mathcal{T}+\mathcal{S} \in L(k(X))$ and $\|\mathcal{T}+\mathcal{S}\| \leq\|\mathcal{T}\|+\|\mathcal{S}\|$

(2) $\alpha \mathcal{T} \in L(k(X))$ and $\|\alpha \mathcal{T}\|=|\alpha|\|\mathcal{T}\|$

(3) $\mathcal{T} \mathcal{S} \in L(k(X))$ and $\|\mathcal{T} \mathcal{S}\| \leq\|\mathcal{T}\|\|\mathcal{S}\|$

Proof. Routine.

Given $T \in L(X)$ we define the map

$$
k(T): k(X) \longrightarrow k(X) \quad, \quad k(T) C:=T C .
$$

Obviously, the restriction of $k(T)$ to $X$ is $T: k(T)\{x\}=T\{x\}=\{T x\}$, for any $x \in X$.

Proposition 3.3. Let $T \in L(X)$. Then $k(T) \in L(k(X))$ and $\|k(T)\|=\|T\|$.

Proof. For $C \in k(X)$, we have that $\|T C\| \leq\|T\|\|C\|$, hence

$$
\|k(T)\|=\sup _{\{0\} \neq C \in k(X)} \frac{\|k(T) C\|}{\|C\|}=\sup _{\{0\} \neq C \in k(X)} \frac{\|T C\|}{\|C\|} \leq\|T\| .
$$

Moreover

$$
\|T\|=\sup _{0 \neq x \in X} \frac{\|T x\|}{\|x\|} \leq \sup _{\{0\} \neq C \in k(X)} \frac{\|T C\|}{\|C\|}=\|k(T)\|
$$

and the proof is completed.

Proposition 3.4. Let $T \in L(X)$. Then $T$ is an isometry if and only if the map $k(T)$ is an isometry.

Proof. It is enough to observe that the equalities

$$
\|k(T) C\|=\|C\|=\|T C\|
$$

are equivalent to that both $k(T)$ and $T$ are isometries.

Our main interest is the study of $(m, q)$-isometries ( $m \geq 1$ integer, $q>0$ real) on the hyperspace $k(X)$. Recall that the general definition was given in (1.1). For $\mathcal{T}: k(X) \longrightarrow k(X)$ the condition (1.1) is equivalent to

$$
\sum_{i=0}^{m}(-1)^{m-i}\left(\begin{array}{c}
m \\
i
\end{array}\right) h\left(\mathcal{T}^{i} C, \mathcal{T}^{i} D\right)^{q}=0 \quad(C, D \in k(X)) .
$$


The equivalence given in Proposition 3.4 can not be extended to $(m, q)$-isometries, although an implication is true.

Proposition 3.5. Let $T \in L(X)$. If the map $k(T)$ is an $(m, q)$-isometry, then $T$ is an $(m, q)$-isometry.

Proof. It is enough to observe that any restriction of an $(m, q)$-isometry to an invariant subset is also an $(m, q)$-isometry and that $T$ is the restriction of $k(T)$ to $X$ as explained before.

The converse of above proposition is false, as we show in the next example.

Example 3.6. Let $S_{w}: \ell_{2} \longrightarrow \ell_{2}$ the weighted shift operator on $\ell_{2}$ with weight sequence $w=\left(w_{n}\right)_{n \geq 1} \in \ell_{\infty}$. That is, for $x=\left(x_{n}\right)_{n \geq 1} \in \ell_{2}$,

$$
S_{w} x=S_{w}\left(x_{1}, x_{2}, x_{3} \ldots\right)=\left(0, w_{1} x_{1}, w_{2} x_{2}, w_{3} x_{3} \ldots\right) .
$$

If $S_{w}$ is a strict $(2,2)$-isometry, then $k\left(S_{w}\right)$ is not a $(2,2)$-isometry.

Proof. We put $\alpha:=\left|w_{1}\right|^{2}$. Then, for $n \geq 1[4$, Remark 3.9(1)(b)]

$$
\left|w_{n}\right|^{2}=\frac{\alpha n-(n-1)}{\alpha(n-1)-(n-2)},
$$

hence

$$
\left|w_{2}\right|^{2}=\frac{2 \alpha-1}{\alpha} \quad \text { and } \quad\left|w_{3}\right|^{2}=\frac{3 \alpha-2}{2 \alpha-1} .
$$

We have that $\alpha \neq 1$ since $S_{w}$ is not an isometry, and $\alpha>1$ since $S_{w}$ is a $(2,2)$ isometry ([4, Remark 3.9(1)(b)], [5, Corollary 2.3]).

Let $\left(e_{n}\right)_{n \geq 1}$ be the canonical basis of $\ell_{2}$. Take $x=e_{1}$ and $y=\lambda e_{2}$, such that $\lambda$ is a scalar with

$$
1<|\lambda|^{2}<\frac{\alpha^{2}}{2 \alpha-1}
$$

We obtain

$$
\begin{gathered}
\|x\|^{2}=1,\left\|S_{w} x\right\|^{2}=\alpha,\left\|S_{w}^{2} x\right\|^{2}=2 \alpha-1 \\
\|y\|^{2}=|\lambda|^{2},\left\|S_{w} y\right\|^{2}=|\lambda|^{2} \frac{2 \alpha-1}{\alpha},\left\|S_{w}^{2} y\right\|^{2}=|\lambda|^{2} \frac{3 \alpha-2}{\alpha} .
\end{gathered}
$$

Consider the segment$$
C=[x, y]:=\{t x+(1-t) y: 0 \leq t \leq 1\} \in k\left(\ell_{2}\right) .
$$

Then

$$
\begin{aligned}
\|C\|^{2} & =\sup _{0 \leq t \leq 1}\|t x+(1-t) y\|^{2} \\
& =\sup _{0 \leq t \leq 1}\|(t,(1-t) \lambda, 0,0,0 \ldots)\|^{2} \\
& =\sup _{0 \leq t \leq 1}\left(t^{2}+(1-t)^{2}|\lambda|^{2}\right) \\
& =|\lambda|^{2}
\end{aligned}
$$


since $1<|\lambda|^{2}$. Moroever,

$$
\begin{aligned}
\left\|S_{w} C\right\|^{2} & =\sup _{0 \leq t \leq 1}\left\|\left(0, w_{1} t, w_{2}(1-t) \lambda, 0,0,0 \ldots\right)\right\|^{2} \\
& =\sup _{0 \leq t \leq 1}\left(\left|w_{1}\right|^{2} t^{2}+\left|w_{2}\right|^{2}(1-t)^{2}|\lambda|^{2}\right) \\
& =\sup _{0 \leq t \leq 1}\left(\alpha t^{2}+\frac{2 \alpha-1}{\alpha}(1-t)^{2}|\lambda|^{2}\right) \\
& =\alpha
\end{aligned}
$$

and

$$
\begin{aligned}
\left\|S_{w}^{2} C\right\|^{2} & =\sup _{0 \leq t \leq 1}\left\|\left(0,0, w_{1} w_{2} t, w_{2} w_{3}(1-t) \lambda, 0,0,0 \ldots\right)\right\|^{2} \\
& =\sup _{0 \leq t \leq 1}\left(\left|w_{1} w_{2}\right|^{2} t^{2}+\left|w_{2} w_{3}\right|^{2}(1-t)^{2}|\lambda|^{2}\right) \\
& =\sup _{0 \leq t \leq 1}\left((2 \alpha-1) t^{2}+\frac{3 \alpha-2}{\alpha}(1-t)^{2}|\lambda|^{2}\right) \\
& =2 \alpha-1 .
\end{aligned}
$$

We have that

$$
\begin{gathered}
h\left(k\left(S_{w}\right)^{2} C, k\left(S_{w}\right)^{2}\{0\}\right)^{2}-2 h\left(k\left(S_{w}\right) C, k\left(S_{w}\right)\{0\}\right)^{2}+h(C,\{0\})^{2}= \\
=\left\|k\left(S_{w}\right)^{2} C\right\|^{2}-2\left\|k\left(S_{w}\right) C\right\|^{2}+\|C\|^{2}=2 \alpha-1-2 \alpha+|\lambda|^{2}=|\lambda|^{2}-1 \neq 0,
\end{gathered}
$$

because of $1<|\lambda|^{2}$. By (3.1) we obtain that $S_{w}$ is not a $(2,2)$-isometry.

\section{The RÅdström SPACE $\widehat{k(X)}$}

Rådström [6] proved that $k(X)$ endowed with the Hausdorff distance can be isometrically embedded in a normed space $\widehat{k(X)}$ in such a way that addition in $\widehat{k(X)}$ induces addition in $k(X)$ and multiplication by scalars in $\widehat{k(X)}$ induces multiplication by scalars in $k(X)$.

Now we give a description of the Rådström space associated to the hyperspace $k(X)$ (see [6]). On $k(X) \times k(X)$ we consider the equivalence relation $(C, D) \sim$ $(E, F) \Longleftrightarrow C+F=D+E$, where $C, D, E, F \in k(X)$. The class of $(C, D)$ is denoted by $[C, D]$.

The quotient space

$$
\widehat{k(X)}:=\frac{k(X) \times k(X)}{\sim}
$$

is a normed space with the following: for $C, D, E, F \in k(X)$ and $\lambda \geq 0$ scalar,

$$
\begin{gathered}
\|[C, D]\|=h(C, D),[C, D]+[E, F]=[C+E, D+F], \\
\lambda[C, D]=[\lambda C, \lambda D],(-\lambda)[C, D]=[\lambda D, \lambda C], .
\end{gathered}
$$

From this, the distance between two classes of $\widehat{k(X)}$ is given by

$$
\widehat{h}([C, D],[E, F])=\|[C, D]-[E, F]\|=\|[C+F, D+E]\|=h(C+F, D+E) .
$$


Moreover the map $\psi: k(X) \longrightarrow \widehat{k(X)}$ defined by $\psi C:=[C,\{0\}]$, is an isometric embedding of $k(X)$ into $\widehat{k(X)}$; in fact, we have that $\psi(C+D)=\psi(C)+\psi(D)$, $\psi(\lambda C)=\lambda \psi(C)$ and $\|\psi(C)\|=\|C\|$.

Given a map $\mathcal{T}: k(X) \longrightarrow k(X)$, we define

$$
\widehat{\mathcal{T}}: \widehat{k(X)} \longrightarrow \widehat{k(X)} \quad, \quad \widehat{\mathcal{T}}[C, D]:=[\mathcal{T} C, \mathcal{T} D] .
$$

Notice that the restriction of $\widehat{\mathcal{T}}$ to $k(X)$ is $\mathcal{T}$.

Proposition 4.1. Let $\mathcal{T}: k(X) \longrightarrow k(X)$ a linear map. Then

(1) $\widehat{\mathcal{T}}$ is linear

(2) $\mathcal{T}$ bounded $\Longrightarrow \widehat{\mathcal{T}}$ bounded and $\|\widehat{\mathcal{T}}\|=\|\mathcal{T}\|$.

Proof. (1) Straightforward.

(2) As $\mathcal{T}$ is restriction of $\widehat{\mathcal{T}}$, we have that $\|\mathcal{T}\| \leq\|\widehat{\mathcal{T}}\|$. Now we show $\|\mathcal{T}\| \geq$ $\|\widehat{\mathcal{T}}\|$. For this purpose, first we prove

$$
h(\mathcal{T} C, \mathcal{T} D) \leq\|\mathcal{T}\| h(C, D) \quad(C, D \in k(X)) .
$$

Fix $C, D \in k(X)$. Let $\varepsilon>h(C, D)$. Then $C \subset D+\varepsilon B_{X}$ and $D \subset C+\varepsilon B_{X}$. Hence $\mathcal{T} C \subset \mathcal{T} D+\varepsilon \widetilde{\mathcal{T}} B_{X}$ and $\mathcal{T} D \subset \mathcal{T} C+\varepsilon \widetilde{\mathcal{T}} B_{X}$, where

$$
\widetilde{\mathcal{T}} B_{X}:=\bigcup_{b \in B_{X}} \mathcal{T}\{b\} .
$$

(Observe that $\mathcal{T} B_{X}$ is not always defined because of $B_{X} \notin k(X)$ if $X$ is infinitedimensional). Notice that from $\mathcal{T}\{b\} \subset\|\mathcal{T}\|\|b\| B_{X} \subset\|\mathcal{T}\| B_{X}$, we obtain $\widetilde{\mathcal{T}} B_{X} \subset$ $\|\mathcal{T}\| B_{X}$ and consequently $\mathcal{T} C \subset \mathcal{T} D+\varepsilon\|\mathcal{T}\| B_{X}$ and $\mathcal{T} D \subset \mathcal{T} C+\varepsilon\|\mathcal{T}\| B_{X}$. Therefore $h(\mathcal{T} C, \mathcal{T} D) \leq \varepsilon\|\mathcal{T}\|$. Hence (4.1) follows. From this

$$
\begin{aligned}
\|\widehat{\mathcal{T}}\| & =\sup _{\|[C, D]\| \leq 1}\|\widehat{\mathcal{T}}[C, D]\| \\
& =\sup _{h(C, D) \leq 1}\|[\mathcal{T} C, \mathcal{T} D]\| \\
& \leq \sup _{h(C, D) \leq 1}\|\mathcal{T}\| h(C, D) \\
& =\|\mathcal{T}\| .
\end{aligned}
$$

So the proof is completed.

Proposition 4.2. Let $\mathcal{T} \in L(k(X))$. The following assertions are equivalent:

(1) $\mathcal{T}$ is a strict $(m, q)$-isometry

(2) $\widehat{\mathcal{T}}$ is a strict $(m, q)$-isometry

Proof. For $C, D \in k(X)$ and $1 \leq k \leq m$, we have the following equalities

$$
\left\|\widehat{\mathcal{T}}^{k}[C, D]\right\|=\left\|\left[\mathcal{T}^{k} C, \mathcal{T}^{k} D\right]\right\|=h\left(\mathcal{T}^{k} C, \mathcal{T}^{k} D\right)
$$


Consequently, $\mathcal{T}$ is an $(m, q)$-isometry, that is it verifies (3.1), if and only if $\widehat{\mathcal{T}}$ verifies

$$
\sum_{i=0}^{m}(-1)^{m-i}\left(\begin{array}{c}
m \\
i
\end{array}\right)\left\|\widehat{\mathcal{T}}^{i}[C, D]\right\|^{q}=0 \quad(C, D \in k(X)) ;
$$

that is, $\widehat{\mathcal{T}}$ is an $(m, q)$-isometry. From this, it is obvious that $\mathcal{T}$ is a strict $(m, q)$ isometry if and only if $\widehat{\mathcal{T}}$ is also a strict $(m, q)$-isometry.

Acknowledgements: The author is partially supported by MTM2013-44357-P (Spain).

\section{REFERENCES}

1. J. Agler, A disconjugacy theorem for Toeplitz operators, Amer. J. Math. 112 (1990), 1-14.

2. F. Bayart, m-isometries on Banach spaces, Math. Nachr. 284 (2011), 2141-2147.

3. T. Bermúdez, A. Martinón and V. Müller, $(m, q)$-isometries on metric spaces, J. Operator Theory 72 (2014), no. 2, 313-329.

4. T. Bermúdez, A. Martinón and E. Negrín, Weighted shift operators which are m-isometries, Integral Equation Operator Theory 68 (2010), 301-312.

5. S.M. Patel. 2-isometric operators, Glasnik Mat. 37 (2002), 143-147.

6. H. Rådström, An Embedding Theorem for Spaces of Convex Sets, Proc. Amer. Math. Soc. 3 (1952), 165-169.

7. O.A.M. Sid Ahmed, m-isometric operators on Banach spaces, Asian-Eur. J. Math. 3 (2010), no. 1, 1-19.

Departamento de Análisis Matemático, Universidad de La Laguna, 38271 LA LAguna (TENERIFE), SPAIN

E-mail address: anmarce@ull.edu.es 